\title{
Novel Optical Fiber Pressure Sensor Using Embedded Fiber Bragg Grating in Acrylonitrile Butadiene Styrene Structure
}

\author{
Chia-Chin Chiang ${ }^{*}$, King-Leung Wong ${ }^{1}$ and Shing-Hai Wang ${ }^{2}$ \\ Department of Mechanical Engineering, National Kaohsiung University of Applied Sciences, \\ 415 Chien Kung Road, Kaohsiung 807, Taiwan \\ ${ }^{1}$ Department of Mechanical Engineering, Kun Shan University No. 195, \\ Kunda Rd., Yongkang Dist., Tainan City 710, Taiwan \\ ${ }^{2}$ Mechanical and Energy Engineering, Kun Shan University No. 195, \\ Kunda Rd., Yongkang Dist., Tainan City 710, Taiwan
}

(Received July 2, 2014; accepted March 26, 2015)

Key words: fiber Bragg grating, acrylonitrile butadiene styrene, pressure sensor, finite element method, stress-strain

In this study, we propose a novel optical fiber pressure sensor based on fiber Bragg grating (FBG). This sensor is simple and has the advantages of being robust and inexpensive. The optical fiber pressure sensor consists of an embedded FBG sensor and an acrylonitrile butadiene styrene (ABS) rectangular cuboid structure. A numerical analysis model of an ABS structure under various pressure loadings is also presented in this paper. A pressure calibration test is carried out to examine the strain variations of the optical fiber pressure sensor at various hydraulic pressures. From the experimental results, the hydraulic pressure is from the $0-10 \mathrm{bar}$; the pressure sensor will change the wavelength conversion with a variable of $380 \mu \varepsilon$. The proposed optical fiber pressure sensor shows a good linear relationship between the water pressure loading and the wavelength shift.

\section{Introduction}

Pressure measurement data is an extremely important aspect of the monitoring system described in this paper. Water pressure changes are an important basis for formation changes and related warnings. However, in addition to being expensive, the conventional electronic pressure gauges currently used bring a number of technical problems that will affect the quality of their measurements. The advantages of fiber Bragg grating (FBG) sensors include the following: they are small, corrosion resistant, and immune to electromagnetic interference. ${ }^{(1)}$ In addition, they can transmit signals over long

*Corresponding author: e-mail: ccchiang@kuas.edu.tw 
distances and perform dynamic real-time monitoring, and FBG has a diameter of about $125 \mu \mathrm{m}$, which is far less than those of the other types of sensor. Moreover, the FBG can also be embedded inside the main components of smart materials, given that structural applications are indispensable. Acrylonitrile butadiene styrene (ABS) is a polymer with high hardness and high chemical and thermal resistance. ${ }^{(2-4)}$ For this reason, in this study, we use an ABS resin material to produce a rectangular cuboid structure as the body of the optical fiber sensor.

\section{Background and Theory}

\subsection{Relevant literature review}

Pressure sensing is particularly important and indispensable as an engineering application. Normally, the most common pressure sensors include strain gauge pressure sensors, piezoresistive pressure sensors, and capacitive pressure sensors. These sensors each have their own disadvantages. For example, these pressure sensors are large, expensive, and show poor resistance to both electromagnetic interference and corrosion. ${ }^{(5)}$ As such, with its high sensitivity, small size, and effective electromagnetic resistance, the optical fiber sensor is more applicable to pressure sensing.(6,7) Zhang et al..$^{(6)}$ and Sheng et al. ${ }^{(7)}$ used similar methods (i.e., the FBG sensor technique) for the production of high-sensitivity pressure sensors to measure pressure. In 2007, Hao et al. ${ }^{(8)}$ conducted a nondestructive evaluation of composite pressure vessels using FBG sensors, but did not simultaneously verify their experimental results by numerical analysis. In 2011, Chena et al. aimed to prove the feasibility of the FBG sensor integrated into a composite tube structure. Uniform FBG sensors were separately embedded in the circumferential and axial directions of a glass-fiber-reinforced plastic tube to detect strain changes under loading. ${ }^{(9)}$ In this study, we combined FBG and ABS resin material to produce an ABS structure at a low cost and to miniaturize the pressure sensors.

\subsection{Principles of FBG sensors}

FBG consists of thousands of short-period refractive index modulations and has a grating period of around $1 \mu \mathrm{m}$. When a broadband light source illuminates the optical fiber and passes through the optical grating, part of the light will be reflected according to the FBG phase matching condition. The light of specific Bragg wavelengths (i.e., light that satisfies the Bragg conditions) will be reflected. The reflected fiber Bragg grating center wavelength can be expressed using the following formula. ${ }^{(10)}$

$$
\lambda_{\mathrm{B}}=2 n_{\mathrm{eff}} \Lambda
$$

Here, $\lambda_{\mathrm{B}}$ is the Bragg wavelength, $n_{\mathrm{eff}}$ is the effective refractive index, and $\Lambda$ is the grating period. When the grating is under strain loading, the drift of the wavelength can be expressed as

$$
\frac{\Delta \lambda}{\lambda}=\left(1-P_{\mathrm{e}}\right) \varepsilon=K_{\varepsilon} \varepsilon
$$


where $\varepsilon$ is strain, $P_{\mathrm{e}}$ is the photoelastic constant, and $K_{\varepsilon}$ is the strain wavelength coefficient. According to the literature, ${ }^{(1)}\left(1-P_{\mathrm{e}}\right)$ is about 0.78 , and when the grating is subjected to tensile strain of $1 \mu \varepsilon$, the Bragg wavelength drift will be about $0.0012 \mathrm{~nm}$.

\section{Study Methodology}

In this study, FBG was used as the pressure-sensing element. This pressure sensor scale had the dimensions of $30(\mathrm{~L}) \times 20(\mathrm{~W}) \times 30(\mathrm{H}) \mathrm{mm}^{3}$, while the internal components used to withstand the pressure had a surface thickness of $2 \mathrm{~mm}$ with 15 $\mathrm{mm}$ as the height of the pressure element. The FBG pressure-sensing elements were fabricated by excimer laser processing shown in Fig. 1.

Figure 2 shows the experimental setup of the static pressure test experiments for the signals emitted by the light source transmitted via the FBG pressure structure, wherein after the light signals are sent to the FBG, they are reflected back along the same optical path of the light source and transmitted to the spectrometer, and the optical signal is then converted to a computer signal for analysis. Finally, Midas software is used for finite element method (FEM) analysis of the pressure sensors under different hydraulic pressures. The software simulation results were compared in order to explore the feasibility of the new pressure sensor developed in this study.

\section{Results and Discussion}

\subsection{Analysis using FEM}

In this study, we simulated a pressure test using FEM. The pressure sensor body was made of the ABS material, and the boundary condition setting section of the original pressure sensor size of $1 / 2$ symmetry plane was selected. The material properties of
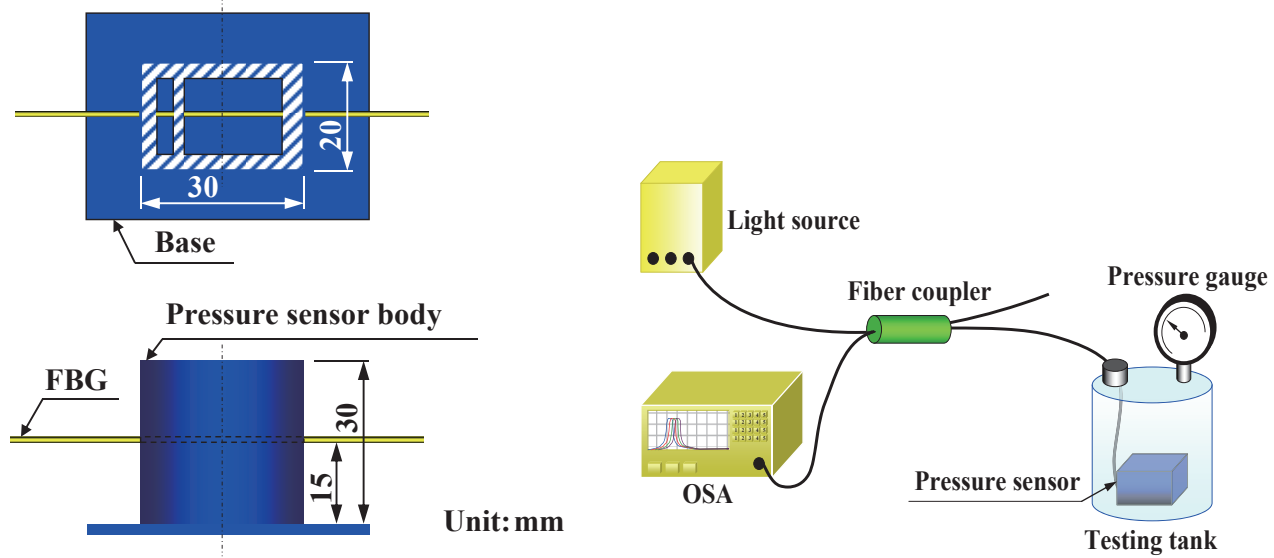

Fig. 1 (left). (Color online) Schematic diagram of the pressure sensors.

Fig. 2 (right). (Color online) Experimental setup of the pressure sensing test. 
ABS and the optical fiber sensor are shown in Table 1. The FEM software (Midas) was used for the hydrostatic test simulation. The FEM model, taking into account the entire group, had 36994 nodes and 127681 elements. The loading boundary of the finite element model is shown in Fig. 3. The simulation results are analyzed and shown in Fig. 3. When exposed to static load of 0-10 bar, the pressure sensor increases the deformation, and stress and strain change linearly, with the pressure brought to bear at the center of the face at high stress concentration. The strain of the FBG under 10 bar loading is shown in Fig. 4.

Table 1

Material properties of ABS and FBG (optical fiber sensor).

\begin{tabular}{lccc}
\hline Material & Young's modulus & Poisson's ratio & Mass density \\
\hline ABS & $127 \mathrm{MPa}$ & 0.275 & $850 \mathrm{~kg} / \mathrm{m}^{3}$ \\
FBG & $73000 \mathrm{MPa}$ & 0.165 & $2.2 \times 10^{6} \mathrm{~kg} / \mathrm{m}^{3}$ \\
\hline
\end{tabular}

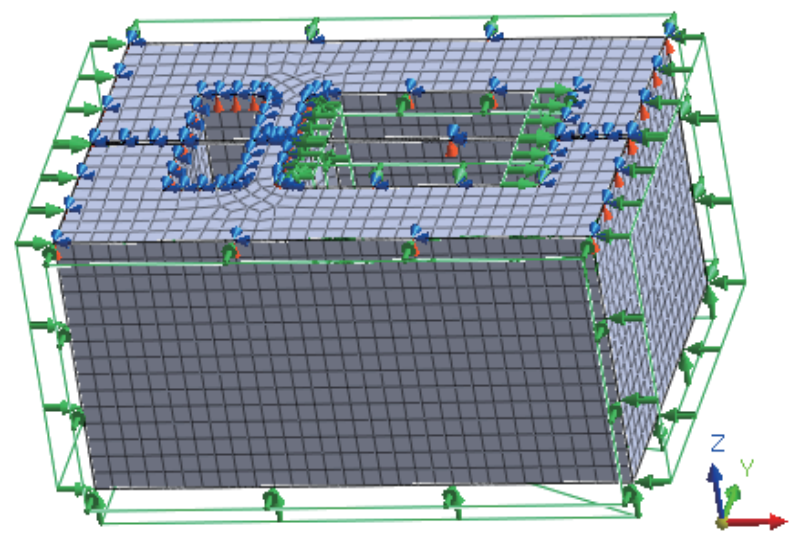

Fig. 3. (Color online) FEM model loading boundary conditions for the pressure sensor body.

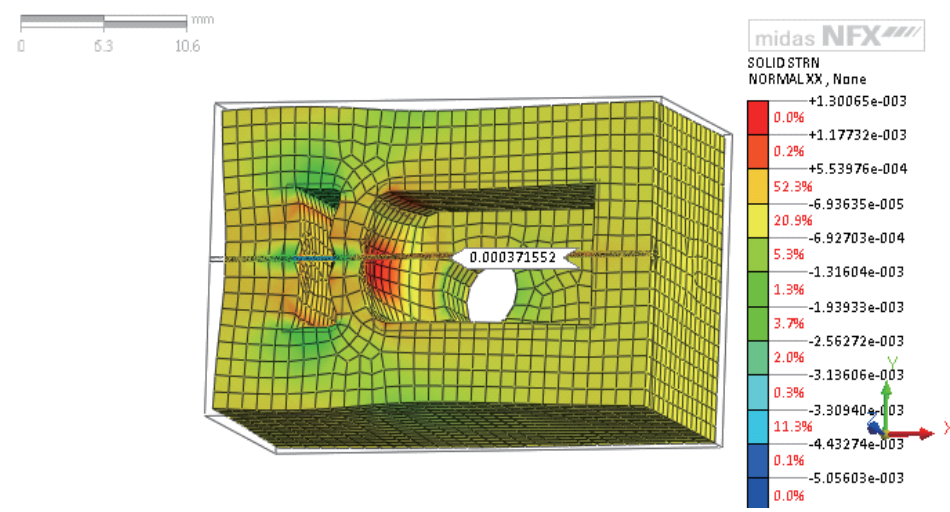

Fig. 4. (Color online) Strain of FBG sensor under 10 bar loading. 


\subsection{Hydrostatic test of the new pressure sensor}

Hydrostatic test results showed that the spectra of the FBG pressure sensor change with various loadings, as shown in Fig. 5. The FBG spectra show linear drifting with pressure loading. From the experimental results, the hydraulic pressure is from 0 to 10 bar, the strain variation is $380 \mu \varepsilon$ under $0-10$ bar pressure loading, while strain variation using the Midas software simulation analysis was also proportional to the pressure loading. The comparison of the simulation and experimental results of the FBG pressure sensor reveals a similar linear relationship, as shown in Fig. 6. The proposed optical fiber pressure sensor shows a good linear relationship between the pressure loading and the wavelength shift. The sensitivity of the pressure sensor was about $38.54 \mu \varepsilon / \mathrm{bar}$.

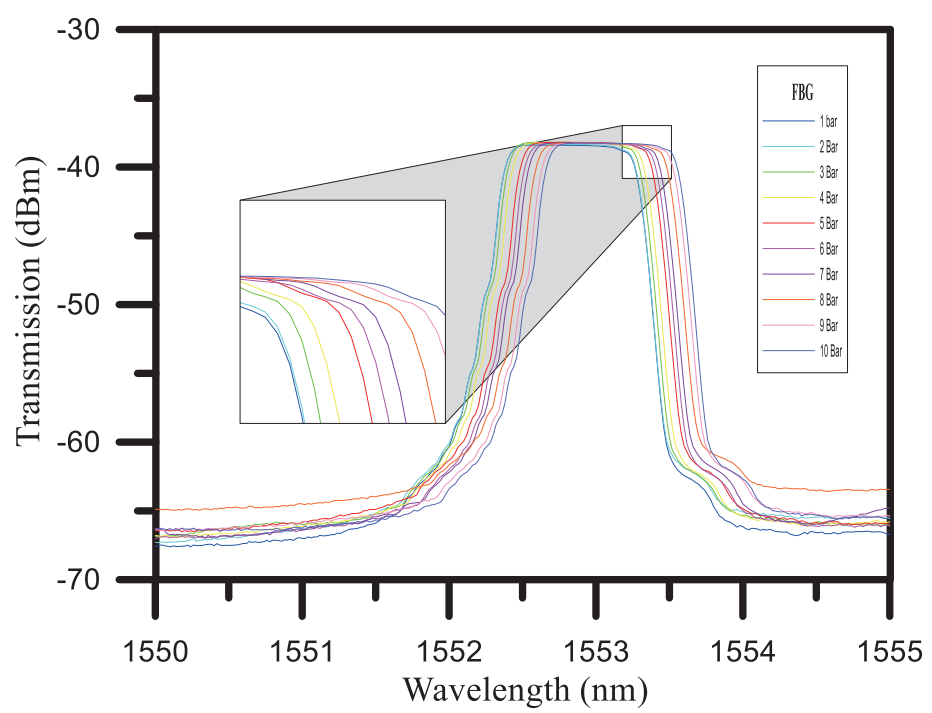

Fig. 5. (Color online) Spectra of the FBG pressure sensor under various loadings.

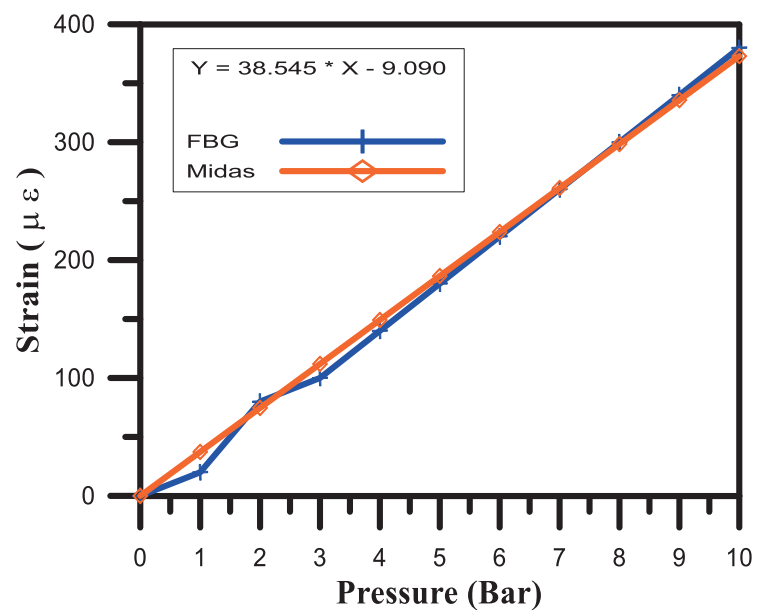

Fig. 6. (Color online) Comparison of the simulation and experimental results of the FBG pressure. 


\section{Conclusions}

In this study, a FBG was used as the pressure-sensing element combined with new technology, resulting in the successful production of a new type of pressure sensor. From a static water pressure experiment, we found a linear relationship between the wavelength of the FBG sensor and the pressure loading when there was deformation of the pressure sensor solid material due to loading from 0 to 10 bar. The sensitivity of the pressure sensor was about $38.54 \mu \varepsilon / b a r$.

\section{Acknowledgements}

This work was funded by the National Science Council, Taiwan (grant number MOST 103-2221-E-151-009-MY3).

\section{References}

1 R. M. Silva, J. M. Baptista, J. L. Santos, A. B. Lobo Ribeiro, F. M. Araújo, L. A. Ferreira and O. Frazão: Opt. Commun. 291 (2013) 215.

2 R. Marissen, D. Schudy, A. V. J. M. Kemp, S. M. H. Coolen, W. G. Duijzings, A. Van Der Pol and A. J. Van Gulick: J. Polym. Sci. 36 (2001) 4167.

3 P. Y. B. Jar, K. Konoshi and T. Shinmura: J. Polym. Sci. 37 (2002) 4521.

4 H. J. Kwon, P.-Y. B. Jar and Z. Xia: J. Polym. Sci. 39 (2004) 4821.

5 J. Y. Joung, K. C. Kim, I. S. Kim and J. Park: Proc. SPIE 4416 (2001) pp. 432-435.

6 Y. Zhang, D. Feng, Z. Liu, Z. Guo, X. Dong, K. S. Chiang and B. C. B. Chu: Photon. Technol. Lett. 13 (2001) 618.

7 H. J. Sheng, M. Y. Fu, T. C. Chen, C. M. Lin, W.-F. Liu and S. S. Bor: Ind. Highway Sens. Technol., SPIE 5272 (2004) 248.

8 J. C Hao, J. S. Leng and Z. Wei: Chinese J. Aeronaut. 20 (2007) 120.

9 Y. C. Chena, C. C. Hsiehb and C. C. Lina: Sens. Actuators, A 167 (2011) 63.

10 M. Majumder, T. K. Gangopadhyay, A. K. Chakraborty, K. Dasgupta and D. K. Bhattacharya: Sens. Actuators, A 147 (2008) 150.

11 R. Kashyap: Principles of Optical Fiber Grating Sensors (Academic Press, Boston, 2009) 2nd ed., Chap. 10, p. 441. 